\title{
An interpretation for the bipolar electric field structures parallel to the magnetic field observed in the auroral ionosphere
}

\author{
Jiankui Shi ${ }^{1}$, M. N. S. Qureshi ${ }^{2}$, K. Torkar ${ }^{3}$, M. Dunlop ${ }^{4}$, Zhenxing Liu ${ }^{1}$, and T. L. Zhang ${ }^{3}$ \\ ${ }^{1}$ State Key Laboratory for Space Weather, CSSAR, Chinese Academy of Sciences, Beijing 100080, China \\ ${ }^{2}$ Department of Physics, Government College University, Lahore 54000, Pakistan \\ ${ }^{3}$ Space Research Institute, Austrian Academy of Sciences, 8042, Graz, Austria \\ ${ }^{4}$ SSTD, Rutherford Appleton Laboratory, Chilton, Didcot, UK
}

Received: 29 August 2007 - Revised: 14 February 2008 - Accepted: 26 March 2008 - Published: 11 June 2008

\begin{abstract}
A physical model for the existence of bipolar structures in the electric field that are parallel to the magnetic field and observed in the auroral ionosphere, is established by deriving the "Sagdeev potential" from the two-fluid equations in a cylindrical coordinate system. The model shows that the bipolar electric field structure can develop not only from an ion acoustic wave, but also from an ion cyclotron wave, when the Mach number and the initial electric field satisfy certain conditions. Moreover, in the auroral region, it shows that the polarity of this electric field structure can be oriented either negative to positive or the reverse polarity, its amplitude can be varied from 35 to $330 \mathrm{mV} / \mathrm{m}$, and its duration can be $7 \mathrm{~ms}$ to $23 \mathrm{~ms}$. These results are in agreement with observation. Therefore, our two-fluid model can interpret the bipolar structures observed in the auroral ionosphere.
\end{abstract}

Keywords. Space plasma physics (Electrostatic structures; Kinetic and MHD theory; Nonlinear phenomena)

\section{Introduction}

The Cluster satellites have observed Electric Field Solitary (EFS) waves, moving parallel to the magnetic field direction, in many space plasma regions, such as the solar wind, magnetosheath, magnetotail, and in the auroral zone (Pickett et al., 2004; Marklund et al., 2004). Indeed, the EFS waves were first observed in the auroral zone by the S3-3 (Temerin et al., 1982) and Viking satellites (Boström et al., 1988). The EFS waves are bipolar structures (one negative peak and one positive peak) moving in the direction parallel to the back-

Correspondence to: M. N. S. Qureshi

(nouman_sarwar25@hotmail.com) ground magnetic field. Mozer et al. (1997), for example, reported a Polar satellite observation of electrostatic solitary waves (i.e. EFS) with amplitudes larger $(\sim 200 \mathrm{mV} / \mathrm{m})$ than any previously reported in the auroral acceleration region, moving along the magnetic field lines at frequencies associated with the proton cyclotron mode. In addition, Bounds et al. (1999) and Dombeck et al. (2001) reported Polar observations of bipolar EFS structures similar to those observed previously by the S3-3 and Viking satellites and inferred the EFS structures to be negative potential pulses traveling upward along the auroral magnetic field lines. Along with ion solitary waves, electron solitary waves have also been observed in the auroral region (Malsov et al., 1993; Ergun et al., 1998; Newmann et al., 2002; Marklund et al., 2004). For the bipolar EFS wave observation, no associated density measurements are reported up to now. But there have been observations of density solitons associated with general electric field solitary structure from the Freja satellite (Dovner et al., 1994; Chen et al., 2000).

For the ion solitary waves, Fig. 1 shows an example of a sequence of bipolar EFS structures observed by Polar in the southern auroral zone at an altitude of $\sim 5478 \mathrm{~km}$ and during a 0.7-s interval after 11:03:41.3 UT on 30 May 1996. One can see that the bipolar structures go from negative to positive with amplitudes up to $140 \mathrm{mV} / \mathrm{m}$ and durations of about $10 \mathrm{~ms}$ while other observations (Ergun et al., 2002) show that the bipolar structures may sometimes go from positive to negative.

Solitary structures in space plasmas have also been studied theoretically by some authors using a two-fluid model. Ion solitary density waves were described as rarefaction ion-acoustic solitary waves and their analysis has been based on a weakly nonlinear theory such as the K-dV (Kortweg-deVries) equation (Washimi and Taniuti,

Published by Copernicus Publications on behalf of the European Geosciences Union. 


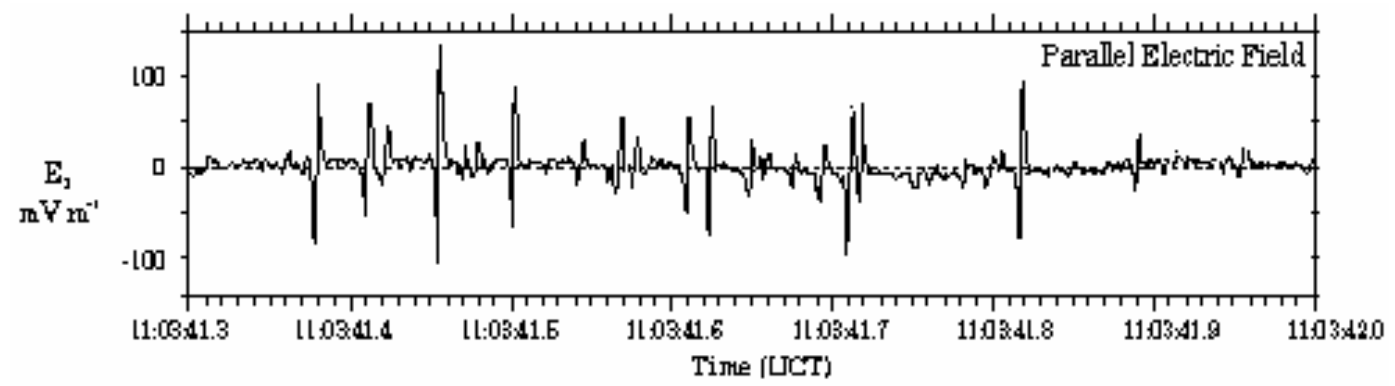

Fig. 1. Example of bipolar electric field pulses parallel to the magnetic field (i.e. z-direction) observed by Polar satellite in the auroral acceleration region (Bounds et al., 1999).

1966). Furthermore, Maxon and Viecelli (1974) studied small-amplitude cylindrically symmetric ion acoustic density waves in the plasma. Shukla and Yu (1978) and Ray (1979) showed that finite amplitude ion acoustic density solitons propagating obliquely to an external magnetic field can also occur. Nonlinear electrostatic density waves propagating nearly perpendicular to the magnetic field have been studied by Chaturvedi (1976) and Temerin et al. (1979) and Lee and Kan (1981) used the exact ion dynamics formulation to show that nonlinear ion-acoustic density waves and solitons propagating obliquely to the magnetic field, can occur in a low- $\beta$ magnetized plasma.

Nonlinear periodic density waves in a cylindrically symmetric magnetic tube have been studied by Molotovshchikov and Ruderman (1987) and Kalita and Bhatta (1994) investigated ion-acoustic solitary density waves, carrying an electron drift, in a warm magnetized plasma. Treumann et al. (1990) investigated a stationary solution for Alfvén solitons (density) propagating obliquely in a homogeneous magnetic field and Wahlund et al. (1994) investigated the relationship between Alfvén wave activity and ion acoustic wave activity within the auroral energization regions. Witt and Lotko (1983) studied ion-acoustic solitary waves, including the electric field that is perpendicular to the magnetic field, in a low- $\beta$ magnetized plasma having an arbitrary electron equation of state. Muschietti et al. $(1999,2002)$ described electron solitary waves as BGK (Bernstein-Greene-Kruskal) electron phase-space holes while Dubouloz et al. (1991) have described them as electron acoustic waves.

In this paper, by considering the structure of bipolar electric field solitary waves, we present a two-fluid model for ions in a low- $\beta$ magnetized plasma with cylindrical symmetry and derive the "Sagdeev potential" from the two-fluid equations. The results from our model show that the bipolar electric field structure propagating along the magnetic field line can develop not only from ion acoustic waves but also from ion cyclotron waves. The results from our model are consistent with observations.

\section{Physical model}

We assume that waves have been excited in a general plasma satisfying ambient conditions as follows:

1. The fluid consists of electrons and ions, and plasma $\beta \ll 1$ which implies that plasma pressure is much smaller than magnetic pressure.

2. The magnetic field is directed along the Z-axis: $\boldsymbol{B}=B_{0} \boldsymbol{e}_{Z}\left(B_{0}\right.$ is constant, $\boldsymbol{e}_{Z}$ is the unit vector along Zaxis). In consequence, we are merely looking for electrostatic solutions and the magnetic field will be passively taken into account in the gyrofrequency.

3. The phase velocity satisfies $v_{T i} \ll v_{p} / \gamma \ll v_{T e}$ (here $v_{\alpha}=\left(2 T_{\alpha} / m_{\alpha}\right)^{1 / 2}$ is the particle thermal velocity, $T_{\alpha}$ and $m_{\alpha}$ are particle energy and mass, subscript " $i$ " denotes the ions and " $e$ " denotes the electrons, $v_{p}$ is phase velocity, $\theta$ is the angle between the wave vector $\boldsymbol{k}$ and $\boldsymbol{e}_{Z}$, and $\gamma=\cos \theta$ ), so that the Landau damping can be neglected.

4. The wave scale $\lambda \gg \lambda_{D}\left(\lambda_{D}\right.$ is the Debye radius), so charge separation effects can be neglected and the quasineutrality condition is may be applied, i.e. $n \approx n_{e} \approx n_{i}(n$ is the particle number density).

Neglecting the electron inertia because the electron mass is much smaller than the ion mass, and using a cylindrical coordinate system, the two-fluid equations for the ions can be written as

$$
\begin{aligned}
& \frac{\partial n}{\partial t}+\frac{\partial\left(n v_{r}\right)}{\partial r}+\frac{\partial\left(n v_{z}\right)}{\partial z}=-\frac{n v_{r}}{r} \\
& \frac{\partial v_{r}}{\partial t}+v_{r} \frac{\partial v_{r}}{\partial r}+v_{z} \frac{\partial v_{r}}{\partial z}=-\frac{1}{m_{i} n} \frac{\partial p}{\partial r}-\frac{e}{m_{i}} \frac{\partial \phi}{\partial r}+\frac{v_{\theta}^{2}}{r}+\Omega_{i} v_{\theta} \\
& \frac{\partial v_{\theta}}{\partial t}+v_{r} \frac{\partial v_{\theta}}{\partial r}+v_{z} \frac{\partial v_{\theta}}{\partial z}=-\frac{v_{r} v_{\theta}}{r}-\Omega_{i} v_{r} \\
& \frac{\partial v_{z}}{\partial t}+v_{r} \frac{\partial v_{z}}{\partial r}+v_{z} \frac{\partial v_{z}}{\partial z}=-\frac{1}{m_{i} n} \frac{\partial p}{\partial z}-\frac{e}{m_{i}} \frac{\partial \phi}{\partial z}
\end{aligned}
$$


with

$p=n T_{i}$

$n=n_{i} \approx n_{e} \approx n_{0} \exp \left(e \phi / T_{e}\right)$,

where $p$ is the thermal pressure, $e$ is the elementary charge, $\phi$ is the electric potential, $v$ is the ion velocity and $n_{0}$ is constant. First, we make a linear approximation for the twofluid Eqs. (1-4). Assuming that a small amplitude disturbance takes place in the background plasma, we linearize the Eqs. (1-4), and obtain the linear dispersion relation as $\omega^{2}\left(\omega^{2}-a C_{s}^{2} k^{2}\right) \quad\left(\omega^{2}-\Omega_{i}^{2}\right)=0$. Here $\omega$ is the wave frequency, the coefficient $a=T_{i} / T_{e}+1, C_{s}=\left(T_{e} / m_{i}\right)^{1 / 2}$ is the ion acoustic velocity, $k$ is wave number, and $\Omega_{i}=e B_{0} /\left(m_{i} c\right)$ is ion gyrofrequency. From dispersion relation, we obtain, $\omega^{2}=a C_{s}^{2} k^{2}$ and $\omega^{2}=\Omega_{i}^{2}$, which show that an ion acoustic wave and an ion cyclotron wave, respectively, can be excited in the plasma described by our model and can probably develop into nonlinear waves.

In order to find the nonlinear solutions of the two-fluid Eqs. (1-6), we introduce the following dimensionless quantities: $N=n / n_{0}, \tau=\Omega_{i} t, R=r / \rho_{i}, Z=z / \rho_{i}, V=v / C_{s}, \Phi=e \phi / T_{e}$, $M=v_{p} / C_{s}, v_{p}=\omega / k$ (here $\rho_{i}$ is the ion gyroradius). We look for solutions of Eqs. (1-6) that depend on $r, \theta, z$, and $t$ through the variable

$S=\left(k_{r} r+k_{z} z-\omega t\right) \Omega_{i} / \omega=(\alpha R+\gamma Z-\tau M) / M$,

where $\alpha=\sin \theta, k_{r}$ and $k_{z}$ are the components of $\boldsymbol{k}$ in the direction of $r$ and $z$. If we consider $K \| \boldsymbol{B}$, i.e. the wave propagation along the magnetic field lines so that, $\alpha=0$ and $\gamma=1$, then from Eqs. (1-7), we can obtain

$$
\begin{aligned}
& -\frac{d N}{d S}+\frac{1}{M} \frac{d\left(N V_{Z}\right)}{d S}=-\frac{N V_{R}}{R} \\
& -\frac{d V_{R}}{d S}+\frac{V_{Z}}{M} \frac{d V_{R}}{d S}=\frac{V_{\theta}^{2}}{R}+V_{\theta} \\
& -\frac{d V_{\theta}}{d S}+\frac{V_{Z}}{M} \frac{d V_{\theta}}{d S}=-\frac{V_{R} V_{\theta}}{R}-V_{R} \\
& -\frac{d V_{Z}}{d S}+\frac{V_{Z}}{M} \frac{d V_{Z}}{d S}=-\frac{a}{M} \frac{d(\ln N)}{d S}
\end{aligned}
$$

where, according to the Eqs. (5) and (6),

$N=\exp (\Phi)$.

By integrating Eq. (11) with the boundary conditions $\left.\boldsymbol{V}\right|_{s=0}=0,\left.N\right|_{s=0}=1$, the $V_{z}$ can be written as

$V_{z}=M\left[1 \pm \sqrt{1-\frac{2 a}{M^{2}} \ln N}\right]$

By substituting Eq. (13) into Eqs. (8-10), we can write Eqs. (8-10), respectively, as

$$
\frac{N V_{R}}{R}= \pm\left(-\sqrt{1-\frac{2 a}{M^{2}} \ln N}+\frac{a}{M^{2}} \frac{1}{\sqrt{1-\frac{2 a}{M^{2}} \ln N}}\right) \frac{d N}{d S}
$$

$$
\begin{aligned}
& \pm \sqrt{1-\frac{2 a}{M^{2}} \ln N} \frac{d V_{R}}{d S}=\frac{V_{\theta}^{2}}{R}+V_{\theta} \\
& \pm \sqrt{1-\frac{2 a}{M^{2}} \ln N} \frac{d V_{\theta}}{d S}=-\frac{V_{\theta} V_{R}}{R}-V_{R} .
\end{aligned}
$$

We now solve the differential Eq. (15) for $V_{\theta}$ and substitute $V_{\theta}$ into Eq. (16) to obtain $d V_{R} / d S$, and then using Eq. (14), we obtain

$$
\begin{aligned}
\frac{d}{d S} & {\left[\frac{1}{N}\left(\frac{a}{M^{2}} \frac{1}{\sqrt{1-\frac{2 a}{M^{2}} \ln N}}-\sqrt{1-\frac{2 a}{M^{2}} \ln N}\right) \frac{d N}{d S}\right] } \\
& =\left(N \sqrt{1-\frac{2 a}{M^{2}} \ln N}-1\right) N .
\end{aligned}
$$

Multiplying the term in brackets ( ) in the left-hand side of the above equation on both sides of Eq. (17), and following the integration by using the boundary condition $\left.\boldsymbol{V}\right|_{s=0}=0, N$ $\left.\right|_{s=0}=1$, we obtain

$\frac{1}{2}\left(\frac{d N}{d S}\right)^{2}+\psi(N)=0$

where

$$
\begin{aligned}
& \psi(N)= \\
& \frac{\left[\left(N \sqrt{1-\frac{2 a}{M^{2}} \ln N}\right)^{2}-\left(\frac{a}{M^{2}}-1\right)^{2} E_{0}^{2}\right]\left(N \sqrt{1-\frac{2 a}{M^{2}} \ln N}\right)^{2}}{2\left[\frac{a}{M^{2}}-\left(1-\frac{2 a}{M^{2}} \ln N\right)\right]^{2}}
\end{aligned}
$$

Here $E_{0}$ is the initial value of $E$ and $E=-1 / N \cdot d N / d S$ according to Eq. (12). Considering Eq. (18), we obtain

$E= \pm \sqrt{-\frac{2}{N^{2}} \psi(N)}$.

Equation (18) is analogous to the energy integral of a classical particle in a 1-D "potential well". $\Psi(N)$ is the so-called the "Sagdeev potential". From Eq. (18), we can analyze the nonlinear plasma density solution and from Eq. (20), we can analyze the electric field structure.

\section{Analysis and discussion}

From Eqs. (18) and (19), solutions for nonlinear waves can be obtained if the parameters lead to a "Sagdeev potential" $\Psi(N)<0$. Our numerical solutions show that the waveform will be different if $\Psi(N)$ has different properties. Here, we choose $S$ as the variable, that means the wave structure is investigated in the coordinate system moving together with the wave.

By analyzing the property of the "Sagdeev potential" $\Psi(N)$, we find that when the plasma parameters satisfy either of the conditions below

$$
\left|\left(a / M^{2}-1\right) E_{o}\right|=1, \quad a / M^{2}<1, \quad G_{m}>2
$$



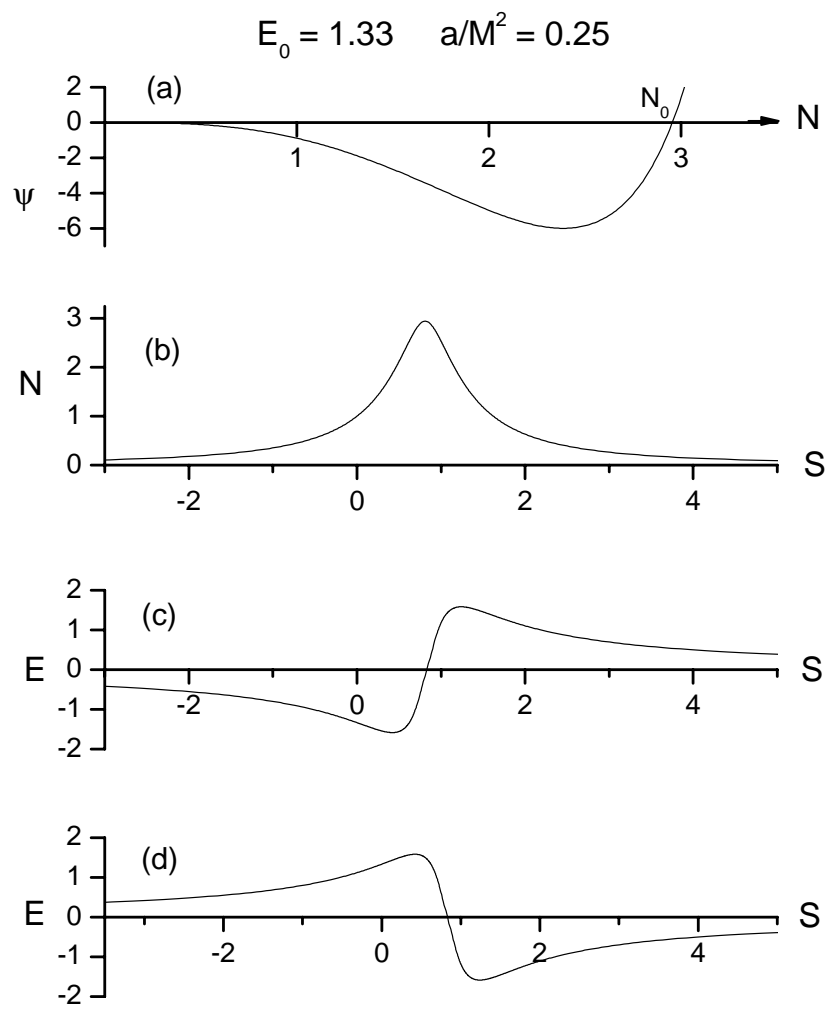

Fig. 2. Profile of "Sagdeev potential", associated density soliton and EFS structures. (a) The "Sagdeev potential" $\Psi(N)$ for the conditions (17-19) is plotted as a function of $N$. (b) Density soliton corresponding to $\Psi(N)$ with $a / M^{2}=0.25$ and $\left|E_{0}\right|=1.33$. (c) Bipolar electric field structure corresponding to the density soliton when $E_{0}<0$. (d) Bipolar electric field structure corresponding to the density soliton when $E_{0}>0$. We chose the magnetic field direction as the $z$-direction (also S direction) in our model.

$\left|\left(a / M^{2}-1\right) E_{o}\right|>1, \quad a / M^{2}<1$

$\left|\left(a / M^{2}-1\right) E_{o}\right|>1, \quad a / M^{2}>1$,

$G_{m} \leq 1+\left|\left(a / M^{2}-1\right) E_{o}\right|$

where

$G_{m}=\sqrt{a / M^{2}} \exp \left[\left(1-a / M^{2}\right) /\left(2 a / M^{2}\right)\right]$

there will be $N_{0}=\exp \left(M^{2} /(2 a)\right)>1$, and $\Psi(N)$ has the properties: $\Psi(0) \rightarrow 0, \Psi\left(N_{0}\right)=0, \Psi^{\prime}(0) \rightarrow 0, \Psi^{\prime}\left(N_{0}\right)>1, \Psi(N)<0$ for $0<N<N_{0}$ (see Fig. 2a).

From Fig. 2a we can see that the $\Psi(N)$ has two zero values at $N=0$ and at $N=N_{0}$, and $\Psi(N)$ is negative between these two points. So, Eq. (18) has a solution corresponding to a density soliton and Eq. (20) has a solution of a bipolar-like EFS. Therefore, Eqs. (21), (22) and (23) are the conditions for the existence of a soliton with a density hump and EFS waves.
Figure $2 \mathrm{~b}$ gives the numerical solution of the typical density soliton wave for $a / M^{2}=0.25$ and $\left|E_{0}\right|=1.33$ under the condition (21). From Fig. 2b, we can see that the normalized density reaches a maximum value of 2.95. Corresponding to Fig. 2b, we can obtain the electric field waveform from Eq. (20) under the same condition (21). Figure 2c shows a typical EFS waveform for the condition (21) when the initial electric field $E_{0}$ is negative. From Fig. 2c, we can see that the electric field has a bipolar structure going from negative to positive and has amplitude of 1.58 . When the initial electric field $E_{0}$ is positive, the EFS waveform for the condition (21) will take a form with opposite polarity, i.e. first positive and then negative (see Fig. 2d).

From Fig. 2b, we should note that the density of the solitary wave gradually approaches zero at the boundary of the solitary wave (i.e. theoretically, $S$ goes to infinity). If the density of the solitary wave along $S$ is integrating from negative infinity to positive infinity in the wave frame, we find that the ion total number remains the same as that before the wave becomes excited. This is conceivable in physics because both ion source and ion sink are not included in our model and the total ion number should be conserved. In fact, the solitary wave is only a localized structure in the vast background medium and its boundaries are still confined within a small area in the background medium but do not cover the whole background medium. In real plasma situation, when the absolute value of $S$ is big enough, the density of the solitary wave will become very small and merge into other disturbance. Therefore, the boundary condition in our model is also conceivable in physics.

Figure 2 only shows a typical example of $\Psi(N)$, the density soliton, and the EFS waveform for the condition (21). For the conditions (22) and (23), the waveform for the density soliton and the electric field structure are similar to that for condition (21). From our model, we can see that an electrostatic density soliton can exist in a low- $\beta$ geo-space plasma with cylindrical symmetry and propagate along the magnetic field when the plasma parameters, Mach number $M$, ratio of ion temperature and electron temperature $a$, as well as the initial electric field $E_{0}$ in the plasma satisfy the conditions (21-23). Moreover, corresponding to the density soliton, the solitary bipolar electric field structures of both polarities "first negative then positive" and "first positive and then negative" moving parallel to the magnetic field can also exist in space plasmas.

Figure 3 depicts how the bipolar EFS structure moving parallel to the local magnetic field could appear in the spacecraft frame. In Fig. 3, the star presents the satellite and the curve presents the bipolar EFS structure. We know that in the polar region the Polar spacecraft is moving almost perpendicular to the magnetic field with a velocity $V_{s}<7.9 \mathrm{~km} / \mathrm{s}$. From our model, we calculated that the bipolar structure moves with a phase speed of $V_{p}>80 \mathrm{~km} / \mathrm{s}$ along the magnetic field and the duration of the bipolar EFS structures lies between 7 to $23 \mathrm{~ms}$ (see next paragraph). Therefore, the satellite speed 
$V_{s}$ would be much less than the phase speed $V_{p}$. The solitary structure has a very short time period of the order of tens of millisecond and moves with a very high speed as compared to the spacecraft, so the spacecraft speed can be neglected. Thus the spacecraft essentially appears to be stationary as the bipolar EFS structure passes along the spacecraft in the wave frame. Hence, the structure will appear as in Fig. 3 or as in the time series data shown in Fig. 1 in the spacecraft frame.

Now let us make a simple comparison between the typical observations and the results from our model for maximum electric field and time duration of the bipolar electric field. From our model, for the wave propagation parallel to the ambient magnetic field, the parameter $S$ in Eq. (7) can be written as $S=\left(k_{z} \cdot z / \omega-t\right) \quad \Omega_{i}=\left(z / v_{p}-t\right) \Omega_{i}$. We take the EFS structure size as $\rho_{i}=C_{s} / \Omega_{i}$, and $M=v_{p} / C_{s}$, the time duration of the EFS structure can be written as $t=(1 / M-S) / \Omega_{i}$. According to the electric field normalization and Eq. (7), the amplitude of electric field can be written as $t=\partial \varphi / \partial z=\left(T_{e} / e\right) \partial(\Phi) / \partial S \cdot \partial S / \partial z$. As $\partial S / \partial z=\Omega_{i} / v_{p}$, we can obtain the electric field as $\mathrm{E}=T_{e} / e \cdot \Omega_{i} /\left(C_{s} M\right) \cdot\left|E_{M}\right|$, where $\left|E_{M}\right|$ is the normalized amplitude of bipolar EFS structures from our model. According to the observations at the orbits of Polar and Fast in the auroral zone, the ion cyclotron frequency $f_{c i}$ is about $100 \mathrm{~Hz}$, the electron temperature ranges from $6 \mathrm{eV}$ to $25 \mathrm{eV}$. Therefore, the $C_{s}$ will range from about $30 \mathrm{~km} / \mathrm{s}$ to $63 \mathrm{~km} / \mathrm{s}$. If we take possible values of $M$ from 2 to $3.3, S$ from 5 to 15 and $\left|E_{M}\right|$ from 1.12 to 1.7 into account, the duration of the bipolar EFS structures will lie between 7 to $23 \mathrm{~ms}$ and their amplitude would be from $35 \mathrm{mV} / \mathrm{m}$ to $330 \mathrm{mV} / \mathrm{m}$. Moreover, the phase speed of the bipolar structures from our model would vary from 60 to $200 \mathrm{~km} / \mathrm{s}$. In particular, for the Fig. $2 \mathrm{c}$ and d, we obtain the duration of the bipolar EFS structure of about $11.8 \mathrm{~ms}$ and an amplitude of about $142 \mathrm{mV} / \mathrm{m}$. This is consistent with the observations in Fig. 1 in particular, where the duration of bipolar structure is about $10 \mathrm{~ms}$ and the electric field amplitude is about $140 \mathrm{mV} / \mathrm{m}$ with phase speed $170 \mathrm{~km} / \mathrm{s}$ (Bounds et al., 1999) and in general, when the duration of the bipolar structure ranges from $2 \mathrm{~ms}$ to $20 \mathrm{~ms}$, the electric field amplitude ranges between $50 \mathrm{mV} / \mathrm{m}$ to $200 \mathrm{mV} / \mathrm{m}$ (Mozer et al., 1997; Bounds et al., 1999; Ergun et al., 2003). Therefore, our two-fluid model can interpret the bipolar structures observed in the auroral ionosphere.

\section{Conclusion}

Bipolar EFS structures observed by various satellites in the auroral region can be formed by the large amplitude ionacoustic and ion-cyclotron waves. These bipolar EFS structures are partially responsible for the acceleration of local plasma to produce magnetic-field-aligned ion-beams that produce discrete auroras and populate the magnetosphere with plasma of ionospheric origin (Mozer et al., 1980). In

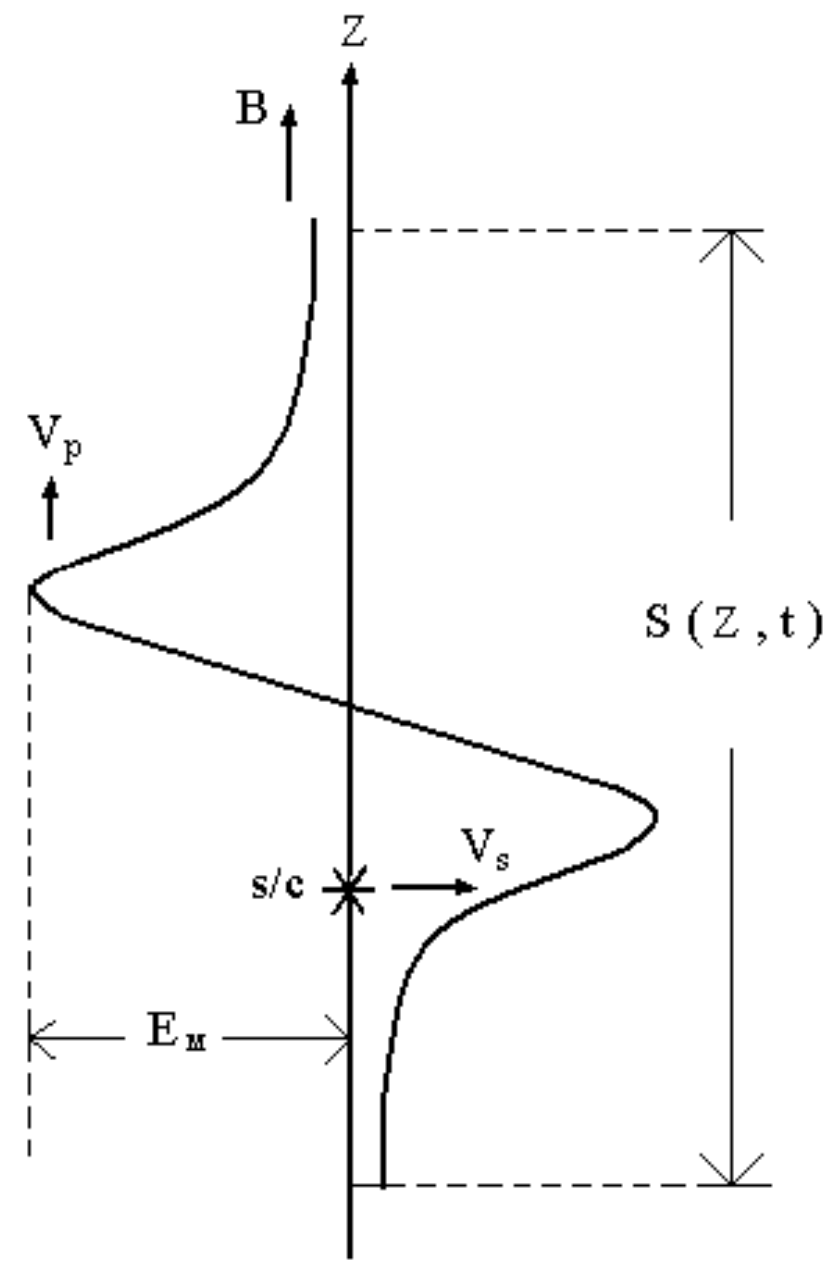

Fig. 3. A carton depicts how the bipolar EFS structure moving parallel to the local magnetic field could appear in the spacecraft frame.

this paper, we consider the three-component ion velocities and proposed a 1-D ion model for the EFS wave propagated along the magnetic field line. Therefore, we could not obtain the transverse size of the wave. But for the wave scale in the field aligned direction, it is easy to be estimated. If we take the phase speed of the bipolar structures from 60 to $200 \mathrm{~km} / \mathrm{s}$ and the duration of the bipolar EFS structures from 7 to $23 \mathrm{~ms}$, the field-aligned size of the wave will be from $0.4 \mathrm{~km}$ to $4.6 \mathrm{~km}$. This is consistent with the observation of ion solitary waves. Furthermore, it should be mentioned that we are merely looking for electrostatic solutions. The magnetic field is passively taken into account in the gyrofrequency. In addition, the required dispersion effect to maintain the nonlinear waves arises from the magnetic force in our study model.

For ion-cyclotron waves its structure must be wider than 2 ion gyroradii $\rho_{i}$. It means $\rho / \rho_{i}>2$ and we can obtain $C_{s} /\left(\Omega_{i} \rho_{i}\right)>2$. If taking $\Omega_{i} \rho_{i}$ as $\left(T_{i} / m_{i}\right)^{1 / 2}$ into account, we can obtain $T_{e} / T_{i}>4$. It implies that the ratio of ion 
temperature to electron temperature should be less than 0.25 . So, we set a restriction on the ion temperature. Furthermore, in our model the condition $v_{T i} \ll v_{p} / \gamma \ll v_{T e}$, is also essential for the EFS waves to exist. It implies that the condition $(2(a-1))^{1 / 2} \ll M \ll\left(2 m_{i} / m_{e}\right)^{1 / 2}$ is satisfied. As the ion mass and the electron mass satisfy $\left(2 m_{i} / m_{e}\right)^{1 / 2} \approx 60$, the ratio of $a-1=T_{i} / T_{e}$ should be small enough to prevent the waves from being affected by Landau damping. This is another restriction on the ion temperature. The two restrictions are consistent.

A two-fluid model is proposed in this paper to interpret the bipolar electric field structures frequently observed by satellites in space plasmas, especially in the auroral region. First, a linear analysis is made for the two-fluid equations to look for mechanisms for the development of a soliton. Then, the "Sagdeev potential" is strictly derived from the two-fluid equations in a cylindrical coordinate system. The results show that EFS waves are bipolar structures and can be of both polarities (first negative then positive, or first positive then negative) depending on the initial value of the electric field $E_{0}$ (negative or positive, respectively). The EFS waves develop not only from ion acoustic waves but also from ion cyclotron waves when the Mach number $M$ and the initial electric field $E_{0}$ satisfy certain conditions. Using the values of the parameters observed by satellites in appropriate positions in the auroral zone for calculation with our model, the amplitude and the duration of the bipolar EFS waves can be from 35 to $330 \mathrm{mV} / \mathrm{m}$ and range from $7 \mathrm{~ms}$ to about $23 \mathrm{~ms}$, respectively. These values are in agreement with observation. Therefore, our model can interpret the EFS waves observed in the auroral region.

Acknowledgements. This research was supported by the National Natural Science Foundation of China under Grant Nos. 40621003 and 40674091, and the CAS International Partnership Programme for Creative Research Teams.

Topical Editor M. Pinnock thanks two anonymous referees for their help in evaluating this paper.

\section{References}

Boström, R., Gustafsson, G., Holback, B., Holmgren, G., Koskinen, H., and Kintner, P.: Characteristics of solitary waves and weak double layers in the magnetospheric plasma, Phys. Rev. Lett., 61, 82-85, 1988.

Bounds, S., Pfaff, R. F., Knowlton, S. F., Mozer, F. S., Temerin, M. A., and Kletzing, C. A.: Solitary potential structures associated with ion and electron beams near 1 Re, J. Geophys. Res., 104, 28709-28 718, 1999.

Chaturvedi, P. K.: Finite amplitude electrostatic ion cyclotron modes, Phys. Fluids, 19, 1064, 1976.

Chen, Y., Li, Z.-Y., Liu, W., and Shi, Z.-D.: Solitary kinetic Alfvén waves in the inertial limit region, Phys. Plasmas, 7, 371-374, 2001.

Dombeck, J., Cattell, C., Crumley, J., Peterson, W., Collin, H., and Kletzing, C.: Observed trends in auroral zone ion solitary wave structure characteristics using data from Polar, J. Geophys. Res., 106, 19013-19022, 2001.

Dovner, P., Eriksson, A., Bostrom, R., and Holback, B.: Freja multiprobe observations of electrostatic solitary structures, Geophys. Res. Lett., 21, 1827-1830, 1994.

Dubouloz, N., Pottelette, R., Malingre, M., and Treumann, R. A.: Generation of broadband electrostatic noise by electron acoustic solitons, Geophys. Res. Lett., 18, 155-158, 1991.

Ergun, R. E., Carlson, C. W., McFadden, J. P., Mozer, F. S., Muschietti, L., Roth, I., and Strangeway, R. J.: Debye-sclae plasma structures associated with magnetic-field-aligned electric fields, Phys. Rev. Lett., 81, 826-829, 1998.

Ergun, R. E., Andersson, L., Main, D. S., Su, Y. J., Carlson, C. W., McFadden, J. P., and Mozer, F. S.: Parallel electric fields in the upward current region of the aurora: Indirect and direct observations, Phys. Plasmas, 9, 3686-3694, 2002.

Ergun, R. E., Andersson, L., Carlson, C. W., Newmann, D. L., and Goldman, M. V.: Debye layers in the downward current region of the aurora, Nonlin. Processes Geophys., 10, 45-52, 2003, http://www.nonlin-processes-geophys.net/10/45/2003/.

Kalita, B. C. and Bhatta, R. P.: Highly nonlinear ion-acoustic solitons in a warm magnetoplasma with drifting effect of electrons, Phys. Plasmas, 1, 2172-2175, 1994.

Lee, L. C. and Kan, J. R.: Nonlinear ion-acoustic waves and solitons in a magnetized plasma, Phys. Fluids, 24, 430-433, 1981.

Marklund, G. T., Karlsson, T., Figueiredo, S., Johansson, T., Lindqvist, P. A., André, M., Buchert, S., Kistler, L. M., and Fazakerley, A.: Characteristics of quasi-static potential structures observed in the auroral return current region by Cluster, Nonlin. Processes Geophys., 11, 709-720, 2004, http://www.nonlin-processes-geophys.net/11/709/2004/.

Maslov, V. and Schamel, H.: Growing electron holes in drifting plasma, Phys. Lett., 78, 171-174, 1993.

Maxon, S. and Vieceli, J.: Krylov-Bogoliubov-Mitropolsky method for nonlinear wave modulation, Phys. Fluids, 17, 1614-1616, 1974.

Molotovshchikov, A. L. and Ruderman, M. S.: Long nonlinear waves in a compressible magnetically structured atmosphere. IV: slow sausage waves in a magnetic tube, Solar Phys., 109, 247 263, 1987.

Mozer, F. S., Cattell, M. K., Hudson, M. K., Lysak, R. L., Temerin, M., and Torbet, R. B.: Satellite measurements and theories of low altitude auroral particle acceleration, Space Sci. Rev., 27, 155-213, 1980.

Mozer, F. S., Ergun, R., Temerin, M., Cattell, C., Dombeck, J., and Wygant, J.: New features of time domain electric-field structures in the auroral acceleration region, Phys. Rev. Lett., 79, 12811284, 1997.

Muschietti, L., Ergun, R. E., Roth, I., and Carlson, C. W.: Phase space electron holes along magnetic field lines, Geophys. Res. Lett., 26, 1093-1096, 1999.

Muschietti, L., Ergun, R. E., Roth, I., Carlson, C. W., and Berthomier, M.: Modeling stretched solitary waves along magnetic field lines, Nonlin. Processes Geophys., 9, 1093-1096, 2002 ,

http://www.nonlin-processes-geophys.net/9/1093/2002/.

Newman, D. L., Goldman, M. V., and Ergun, R. E.: Evidence for correlated double layers, bipolar structures, and very-lowfrequency saucer generation in the auroral ionosphere, Phys. 
Plasmas, 9, 2337-2343, 2002.

Pickett, J. S., Chen, L. J., Kahler, S. W., Santolik, O., Gurnett, D. A., Tsurutani, B. T., and Balogh, A.: Isolated electrostatic structures observed throughout the Cluster orbit: relationship to magnetic field strength, Ann. Geophys., 22, 2515-2523, 2004,

http://www.ann-geophys.net/22/2515/2004/.

Ray, D.: Exact solitary ion acoustic waves in a magnetoplasma, Phys. Fluids, 22, 2037-2038, 1979.

Shukla, P. K. and Yu, M. Y.: Exact solitary ion acoustic waves in a magnetoplasma, J. Math. Phys., 19, 2506-2508, 1978.

Temerin, M., Woldorff, M., and Mozer, F. S.: Nonlinear Steepening of the Electrostatic Ion Cyclotron Wave, Phys. Rev. Lett., 43, 1941-1943, 1979.

Temerin, M., Cerny, K., Lotko, W., and Mozer, F. S.: Observations of double layers and solitary waves in the auroral plasma, Phys. Rev. Lett., 48, 1175-1179, 1982.
Treumann, R. A., Gudel, M., and Benz, A. O.: Alfvén wave solitons and solar intermediate drift bursts, Astron. Astrophys., 236, 242 249, 1990.

Wahlund, J. E., Louarn, P., Chust, T., de-Feraudy, H., Roux, A., Holback, B., Dovner, P., and Holmgren, G.: On ion acoustic turbulence and the nonlinear evolution of kinetic Alfvén waves in Aurora, Geophys. Res. Lett., 21, 1831-1834, 1994.

Washimi, H. and Taniuti, T.: Propagation of ion acoustic solitary waves of small amplitude, Phys. Rev. Lett., 17, 996-998, 1966.

Witt, E. and Lotko, W.: Ion-acoustic solitary waves in a magnetized plasma with arbitrary electron equation of state, Phys. Fluids, 26, 2176-2185, 1983. 